Check for updates

Cite this: RSC Adv., 2019, 9, 5295

Received 24th November 2018 Accepted 24th January 2019

DOI: 10.1039/c8ra09671f

rsc.li/rsc-advances

\section{Polyphenols extracted from huangjiu have anti- inflammatory activity in lipopolysaccharide stimulated RAW264.7 cells $\dagger$}

\author{
Lin Peng, (D) acd Aisikaer Ai-lati, ${ }^{\text {acd }}$ Zhongwei Ji, ${ }^{\text {acd }}$ Shuguang Chen*b \\ and Jian Mao*acde
}

In the present study, an extraction method, combining extraction by ethyl acetate + ethanol and purification by HPD400 resin, was established to obtain huangjiu polyphenol extract (HPE). After extraction and purification, the polyphenol yield was $22.57 \%$, and $90.57 \%$ protein and $97.99 \%$ sugar were removed. HPLC analysis indicated that $(+)$-catechin $\left(91.33 \mu \mathrm{g} \mathrm{mL}{ }^{-1}\right)$ was the predominant phenolic compound among the 11 detected polyphenols. In LPS-stimulated RAW264.7 cells, HPE exhibits anti-inflammatory effects by inhibiting the production of NO and pro-inflammatory cytokines (TNF- $\alpha$, interleukin IL- 6 and IL-1B). The anti-inflammatory effect of HPE is associated with the inhibition of iNOS expression, the suppression of NF- $\kappa B$ translocation to the nucleus, and the inhibition of the phosphorylation of $1 \kappa B$ and the MAPK family proteins, e.g. p-38, Erk 1/2, and JNK. Moreover, the activation of Nrf2 and HO-1 is also related to the anti-inflammatory effect of HPE.

\section{Introduction}

Huangjiu, also named Chinese rice wine, is a traditional alcoholic beverage and has a long history in China. Huangjiu is produced by following a traditional and unique process. ${ }^{1}$ It is fermented from steamed glutinous rice mixed with wheat $\mathrm{Qu}$ (as the saccharifying agent) and yeast (as the fermentation starter). After 3-5 days of fermentation at $28-32{ }^{\circ} \mathrm{C}$, the fermentation mash carries out post-fermentation for approximately 1 month. Fresh huangjiu is separated from vinasse by filtration and further clarified by membranes and diatomite. After sterilization by thermal treatment, the maturation of fresh huangjiu is carried out by being sealed in pottery jars for more than 3 years. In China, huangjiu has several varieties according to different geographical origins. These regions can be divided into the eastern, central, and southern parts of China, including the Zhejiang, Hubei, Yunnan, Jiangsu, and Sichuan provinces.

\footnotetext{
${ }^{a}$ National Engineering Laboratory for Cereal Fermentation Technology, Jiangnan University, Wuxi, 214122, China.E-mail: maojian@jiangnan.edu.cn

${ }^{b}$ Department of General Surgery, Peking Union Medical College Hospital, Chinese Academy of Medical Sciences and Peking Union Medical College (CAMS \& PUMC), Beijing, 100730, China.E-mail: cshuguang6@sina.com

${ }^{c}$ School of Food Science and Technology, Jiangnan University, Wuxi, Jiangsu 214122, China

${ }^{d}$ National Engineering Research Center of Chinese Rice Wine, Shaoxing, Zhejiang 31200, China

${ }^{e}$ State Key Laboratory of Food Science \& Technology, Jiangnan University, Wuxi, Jiangsu 214122, China
}

† Electronic supplementary information (ESI) available. See DOI: 10.1039/c8ra09671f
The diversity of the geographical origins leads to the variety of bioactive constituents in huangjiu. ${ }^{2}$

According to current research, huangjiu has been found to possess pharmacological and nutritional benefits, which can be attributed to the various bioactive constituents in huangjiu. ${ }^{\mathbf{3} 4}$ These functional and nutritional compounds include polysaccharides, ${ }^{5}$ peptides, ${ }^{6}$ amino acids,${ }^{7}$ mineral elements, ${ }^{8} \gamma$-aminobutyric acid, ${ }^{9}$ hydroxymethylfurfural, ${ }^{10} 4$-vinylguaiacol, ${ }^{11}$ etc. In huangjiu, the formation of polyphenols is related to the raw materials and wheat Qu during huangjiu fermentation. Glutinous rice contains several bioactive compounds (such as amino acids and polyphenols), and these compounds are transferred to huangjiu during fermentation. The composition and content of the phenolic compounds in huangjiu are related to the rice used during fermentation. ${ }^{12,13}$ Moreover, some polyphenols are also regarded as aroma compounds and the concentration of polyphenol can be improved by the addition of wheat Qu, compared with commercial enzymes used as saccharifying agents. ${ }^{14}$

Polyphenols are commonly divided into two main groups: flavonoids and non-flavonoids, but there are many more than four classes. ${ }^{15}$ Phenolic compound analysis of different varieties of huangjiu showed a great difference in the composition and content. ${ }^{16,17}$ Zhang et al. ${ }^{18}$ demonstrated that huangjiu has antioxidant activities, including reducing capacity and radical scavenging activity. These antioxidant activities are highly related to the total phenolic content in huangjiu. ${ }^{13}$ It has been implicated that polyphenolic compounds can be preventive and therapeutic agents for the treatment of various diseases due to their antioxidant properties. ${ }^{19-21}$ In a previous study, ${ }^{22}$ huangjiu showed great health benefits for cardiovascular disease in 
mouse models with plasma hyperhomocysteinemia and atherosclerosis. After feeding with huangjiu, the plasma homocysteine $(p<0.01)$ and atherosclerosis lesion areas $(p<$ 0.001 ) of the model mice were significantly reduced compared to the control and ethanol groups. Further work from the same group found that polyphenolic compounds extracted from huangjiu have anti-atherosclerotic activity by inhibiting matrix metalloproteinase (MMP)-2 and MMP-9. ${ }^{23}$ As shown through in vitro experiments, huangjiu polyphenol extract also exhibited inhibitory effects on the differentiation ${ }^{24}$ and homocysteine induced proliferation and migration ${ }^{25}$ of vascular smooth muscle cells. As well as anti-atherosclerotic activity, polyphenolic compounds extracted from some fruits and edible plants exhibit great anti-inflammatory activity, which is related to antioxidant activity. ${ }^{26-28}$ However, little attention has been paid to the anti-inflammatory activity of polyphenols extracted from huangjiu, despite their great antioxidant activity.

Therefore, we established an extraction method to effectively separate polyphenolic compounds from huangjiu. After analysing the composition profile, the anti-inflammatory activity of huangjiu polyphenol extract (HPE) was investigated in lipopolysaccharide (LPS) stimulated RAW264.7 cells by detecting the production of nitric oxide and pro-inflammatory cytokines. Moreover, the expression of some proteins relating to inflammatory activity was also analysed to gain a better understanding of the possible mechanism.

\section{Materials and methods}

\section{Chemicals}

RPMI-1640 medium and fetal bovine serum (FBS) were purchased from Gibco Co. (Carlsbad, CA). Dimethyl sulfoxide (DMSO), lipopolysaccharide from Escherichia coli, 3-(4,5dimethyl-2-thizolyl)-2,5-diphenyl-2 $H$-tetrazolium bromide (MTT), Griess reagent, penicillin and streptomycin were purchased from Sigma Co. (St Louis, MO). An enzyme-linked immunosorbent assay kit (Elisa kit) for TNF- $\alpha$, IL-1 $\beta$ and IL-6 detection was purchased from Sigma Co. The standards for phenolic acid detection were obtained from Sigma Co. RIPA lysis buffer was purchased from Biosharp Co. (Anhui, China). A BCA kit for protein concentration detection was purchased from Beyotime Co. (Shanghai, China). The antibodies used in this study for inducible nitric oxide synthase (iNOS), heme

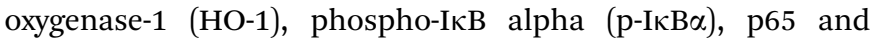
nuclear factor erythroid-2-related factor 2 (Nrf2) were purchased from Cell Signaling Technology (Beverly, MA). Antibodies for the MAPK family (normal and phosphorylated (P-) proteins), including p-38, P-p38, Erk 1/2, P-Erk 1/2, JNK and P-JNK, were purchased from cell signaling technology. Anti $\beta$-actin antibodies were purchased from Sigma Co. Horseradish peroxidase (HRP)-conjugated anti-goat and anti-rabbit IgG were purchased from Cell Signaling Technology.

\section{Polyphenol extraction and purification}

To remove polysaccharides and proteins, huangjiu purchased from Guyuelongshan Co. (Zhejiang, China) was mixed with ethanol in the ratio of $1: 4(\mathrm{v} / \mathrm{v})$ and the mixture was stored at $4{ }^{\circ} \mathrm{C}$ overnight. After centrifugation $\left(10000 g, 4{ }^{\circ} \mathrm{C}\right)$ for $30 \mathrm{~min}$, the supernatant was mixed with the same volume of ethyl acetate. The mixture was stirred at $4{ }^{\circ} \mathrm{C}$ for $30 \mathrm{~min}$, and then centrifuged for $20 \mathrm{~min}\left(10000 \mathrm{~g}, 4^{\circ} \mathrm{C}\right)$. The supernatant, filtered by a $0.45 \mu \mathrm{m}$ fluoroethylene filter, was evaporated until dry under vacuum at $40{ }^{\circ} \mathrm{C}$ and then the crude extract was dissolved in $0.5 \%$ DMSO solution at a concentration of $1 \mathrm{mg}$ gallic acid equivalent (GAE) per mL, which was analysed according to the Folin-Ciocalteu method. ${ }^{29}$ Twenty five millilitres of polyphenol solution was further absorbed by $10 \mathrm{~g}$ prepared macroporous HPD-400 resin (Mitsubishi Chemical Holding, Tokyo, Japan). After washing by $0.5 \%$ DMSO solution, the absorbed polyphenols were eluted by $10 \mathrm{~mL}$ ethanol and stored at $-20{ }^{\circ} \mathrm{C}$ for further study. The entire extraction and purification process is summarized in Fig. S1 (ESI $\dagger$ ). The total sugar content was analysed by a phenol-sulfuric acid assay. The total nitrogen content was analysed by Kjeldahl determination and calculated using a constant of 6.25 .

\section{HPLC analysis of the polyphenols in huangiiu extract}

HPLC analysis for the polyphenols was carried out by a Waters $1525 \mathrm{EF}$ with a ZORAX SB-C18 reversed-phase column $(4.6 \mathrm{~mm}$ $\times 150 \mathrm{~mm}, 5 \mu \mathrm{m}$ particle size), which was protected by a guard column. The sample $(50 \mu \mathrm{L})$ was filtrated and the injection volume was $20 \mu \mathrm{L}$. The absorbance wavelength for polyphenols was scanned at $280 \mathrm{~nm}$. The mobile phase used in the HPLC analysis was composed of solution A (5\% methanol and $0.05 \%$ $\left.\mathrm{H}_{3} \mathrm{PO}_{4}\right)$ and solution $\mathrm{B}\left(80 \%\right.$ methanol and $\left.0.05 \% \mathrm{H}_{3} \mathrm{PO}_{4}\right)$. The procedures of gradient elution were: $0 \mathrm{~min}(95 \% \mathrm{~A}+5 \% \mathrm{~B})$, $30 \min (35 \% \mathrm{~A}+65 \% \mathrm{~B}), 40 \mathrm{~min}(0 \% \mathrm{~A}+100 \% \mathrm{~B}), 45 \mathrm{~min}(0 \%$ $A+100 \% \mathrm{~B}), 52 \min (95 \% \mathrm{~A}+5 \% \mathrm{~B})$, and $58 \min (95 \% \mathrm{~A}+5 \% \mathrm{~B})$ with a flow rate of $0.6 \mathrm{~mL} \mathrm{~min}^{-1}$ at $30{ }^{\circ} \mathrm{C}$. The polyphenols in HPE were identified by comparing the retention time of the standards and the content was calculated by calibration curves obtained from the linear correlation between various concentrations of the standards and peak area.

\section{NO and pro-inflammatory cytokine determination}

Murine macrophage cell line RAW264.7 was purchased from the Chinese Academy of Sciences (Shanghai, China). Cells were seeded at a density of $1 \times 10^{4}$ cells per well into a 96-well microplate. After culturing at $37^{\circ} \mathrm{C}$ for $24 \mathrm{~h}$ in a $\mathrm{CO}_{2}$ incubator, cells were pre-treated with various concentrations $(100,50,25$, and $0 \mu \mathrm{g} \mathrm{mL} \mathrm{m}^{-1}$ ) of HPE for $4 \mathrm{~h}$ and then stimulated by $0.1 \mu \mathrm{g}$ $\mathrm{mL}^{-1}$ LPS. Cells without the treatment of LPS and HPE were used as a control. The cell medium supernatant was withdrawn to analyse the concentration of nitric oxide, TNF- $\alpha$, IL-1 $\beta$ and IL6 . Fifty $\mu \mathrm{L}$ supernatant and various concentrations of sodium nitrite $(80-2.5 \mu \mathrm{M})$ were mixed with $50 \mu \mathrm{L}$ Griess reagent. The mixture was incubated at room temperature for $30 \mathrm{~min}$, and the absorbance at $540 \mathrm{~nm}$ was measured to calculate the nitric oxide concentration. Cell free medium was used as a control. The concentrations of different pro-inflammatory cytokines, including TNF- $\alpha$, IL-1 $\beta$ and IL-6, were analysed by commercial Elisa kits according to the manufacturers' instructions. 


\section{Western blot}

RAW264.7 cells were seeded in 6-well plates at a density of $2 \times$ $10^{5}$ cells per well and treated with LPS and HPE as mentioned above. The cells in the plates were washed by PBS solution two times and suspended by RIPA lysis buffer with a protease inhibitor. The protein concentration in the obtained cell lysates was detected by a BCA kit. The proteins in the lysates were separated by $10 \%$ sodium dodecyl sulfate-polyacrylamide gel electrophoresis (SDS-PAGE) and then transferred to polyvinylidene difluoride (PVDF) membranes. The membranes were blocked by $5 \%$ skim milk, and sequentially treated with primary antibody and horseradish peroxidase-conjugated anti-goat or anti-rabbit IgG. The protein bands were visualized and the densities of the bands were quantified by the software Image J.

\section{Statistical analysis}

All of the data in this study were shown as a means of \pm SEM. Statistical analysis was carried out by the GraphPad Prism 6.0 software. The levels of statistical significance between data sets were defined as: significant if $p<0.05\left(^{*}\right)$, and highly significant if $p<0.01(* *)$.

\section{Results}

\section{Extraction and HPLC analysis of huangjiu polyphenol extract}

In the present study, a method combining extraction and purification was established to obtain huangjiu polyphenol extract (HPE) and the procedures are shown in Fig. S1 (ESI $\dagger$ ). During extraction, polysaccharides and proteins in huangjiu were removed by precipitation with $80 \%(\mathrm{v} / \mathrm{v})$ ethanol, and the polyphenols were extracted following the addition of ethyl acetate. The organic phase was concentrated by rotary evaporation and the crude polyphenol extract was dissolved in $0.5 \%$ DMSO solution for further purification with HPD400 resin. Finally, huangjiu polyphenol extract (HPE) was eluted by ethanol and a polyphenol yield of $22.57 \%$ was achieved (ESI Table S1 $\dagger$ ). Moreover, this method effectively removed the total protein and the total sugar, and the removal rates were $90.57 \%$ for the total protein and $97.99 \%$ for the total sugar, respectively. The HPE obtained by this method was used to analyse the polyphenol composition by HPLC and to test the antiinflammatory activity in RAW264.7 cells. HPLC analysis (shown in ESI Fig. S2 $\dagger$ ) indicated that the HPE contained 11 polyphenols, including protocatechuic acid $\left(0.3 \mu \mathrm{g} \mathrm{mL} \mathrm{mL}^{-1}\right)$, (+)-catechin $\left(91.33 \mu \mathrm{g} \mathrm{mL}{ }^{-1}\right)$, chlorogenic acid $\left(5.95 \mu \mathrm{g} \mathrm{mL}{ }^{-1}\right)$, vanillic acid $\left(1.85 \mu \mathrm{g} \mathrm{mL}{ }^{-1}\right)$, caffeic acid $\left(1.21 \mu \mathrm{g} \mathrm{mL}^{-1}\right)$, syringic acid $\left(0.37 \mu \mathrm{g} \mathrm{mL}{ }^{-1}\right), p$-coumaric acid $\left(2.25 \mu \mathrm{g} \mathrm{mL}{ }^{-1}\right)$, ferulic acid $\left(2.97 \mu \mathrm{g} \mathrm{mL}{ }^{-1}\right)$, sinapic acid $\left(1.93 \mu \mathrm{g} \mathrm{mL}^{-1}\right)$, rutin $(0.84 \mu \mathrm{g}$ $\left.\mathrm{mL}^{-1}\right)$ and quercetin $\left(2.15 \mu \mathrm{g} \mathrm{mL}^{-1}\right)$ (Table 1). Among these detected polyphenols, (+)-catechin was the predominant phenolic compound in the HPE.

Effect of HPE on the production of NO and pro-inflammatory cytokines in LPS stimulated RAW264.7 macrophages

As shown in Fig. S3 (ESI $\dagger$ ), HPE had no negative effect on the cell viability of RAW264.7 macrophages at concentrations below
Table 1 Composition and content of the polyphenols in huangjiu polyphenol extract (HPE)

\begin{tabular}{lcc}
\hline Compounds & Retention time $(\mathrm{min})$ & Contents $\left(\mu \mathrm{g} \mathrm{mL}^{-1}\right)$ \\
\hline Protocatechuic acid & 10.89 & $0.3 \pm 0.02$ \\
$(+)$-Catechin & 14.60 & $91.33 \pm 8.64$ \\
Chlorogenic acid & 15.90 & $5.95 \pm 0.37$ \\
Vanillic acid & 17.85 & $1.85 \pm 0.13$ \\
Caffeic acid & 18.12 & $1.21 \pm 0.27$ \\
Syringic acid & 19.23 & $0.37 \pm 0.03$ \\
p-Coumaric acid & 22.93 & $2.25 \pm 0.12$ \\
Ferulic acid & 24.04 & $2.97 \pm 0.31$ \\
Sinapic acid & 24.39 & $1.93 \pm 0.08$ \\
Rutin & 27.63 & $0.84 \pm 0.09$ \\
Quercetin & 34.12 & $2.15 \pm 0.22$
\end{tabular}

$100 \mathrm{mg} \mathrm{GAE} / \mathrm{L}$. Therefore, HPE with concentrations of $0,25,50$, and $100 \mathrm{mg}$ GAE/L were used in subsequent anti-inflammatory activity investigations. To investigate the inflammatory activity of HPE, the production of NO was analysed after incubating with various concentrations of HPE and $0.1 \mu \mathrm{g} \mathrm{mL}{ }^{-1}$ LPS. The results showed that NO production was effectively promoted by LPS treatment (Fig. 1a). To further elucidate the inhibitory effect of HPE on NO production, iNOS expression during LPS and HPE treatment was carried out by western blotting in this study. The results showed that the expression of iNOS was obviously improved by LPS treatment, and incubation with various concentrations of HPE downregulated the expression of iNOS (Fig. 1b). As well as NO production, LPS treatment also significantly increased the production of pro-inflammatory cytokines (TNF- $\alpha$, IL-6 and IL-1 $\beta$ ). Meanwhile, incubation with HPE hardly enhanced pro-inflammatory cytokine production in the RAW264.7 cells without LPS treatment (Fig. 2), indicating no

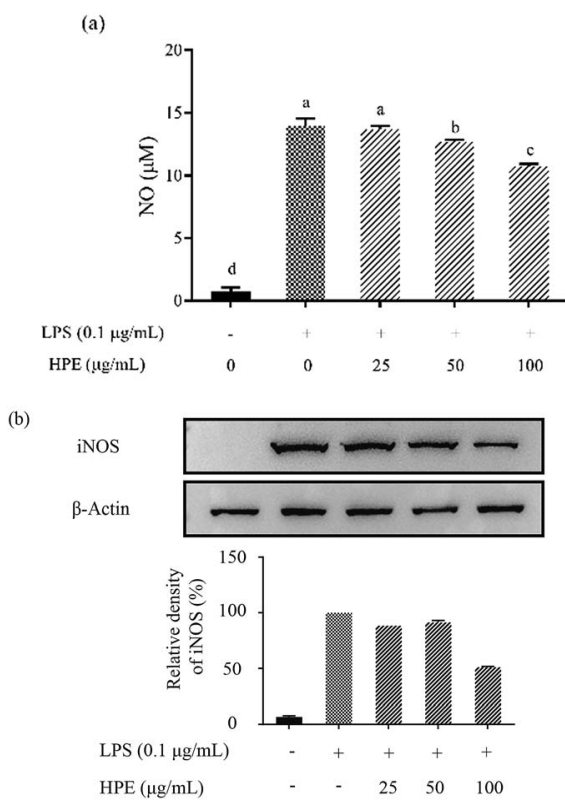

Fig. 1 Effect of huangjiu polyphenol extract (HPE) on the production of (a) NO and (b) the expression of iNOS in LPS-stimulated RAW264.7 macrophages. 

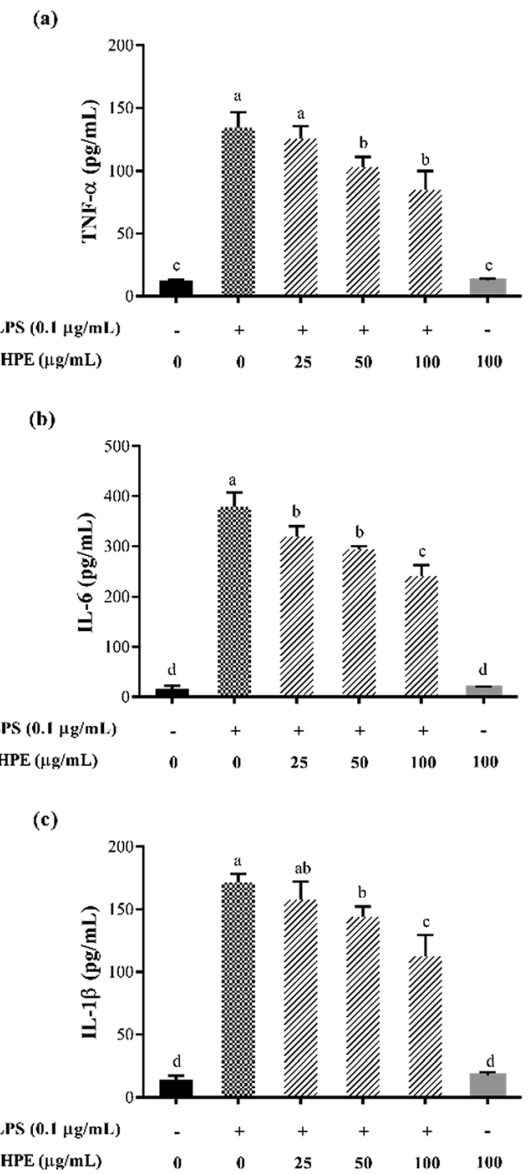

Fig. 2 Effect of huangjiu polyphenol extract (HPE) on the production of (a) TNF- $\alpha$, (b) IL- 6 and (c) IL-1 $\beta$ in RAW264.7 macrophages with or without LPS stimulation.

induced effect of the HPE on inflammation. Incubation with HPE (especially at a concentration of $100 \mu \mathrm{g}$ GAE per mL) effectively downregulated the production of these LPS-induced pro-inflammatory mediators (Fig. 2). From these results, we found that HPE efficiently reduces the production of pro-inflammatory cytokines in LPS-stimulated RAW264.7 cells.

\section{Effect of HPE on Nrf2 translocation and HO-1 expression in LPS stimulated RAW264.7 macrophages}

After WB analysis, the results showed that the translocation of Nrf2 from the cytoplasm to the nucleus was significantly inhibited by LPS treatment in RAW264.7 macrophages (Fig. 3a). Similarly, HO-1 expression in the RAW264.7 cells was also suppressed by LPS treatment (Fig. 3b). However, Nrf2 in the cytoplasm was effectively reduced after pre-treating HPE, indicating the up-regulation of Nrf2 translocation. Meanwhile, HO-1 expression in the RAW264.7 cells was also upregulated after treating HPE, especially at higher concentrations. Therefore, the Nrf2/HO-1 pathway plays an important role in the down-regulation of pro-inflammatory cytokine production in HPE treated LPS stimulated RAW264.7 macrophages.
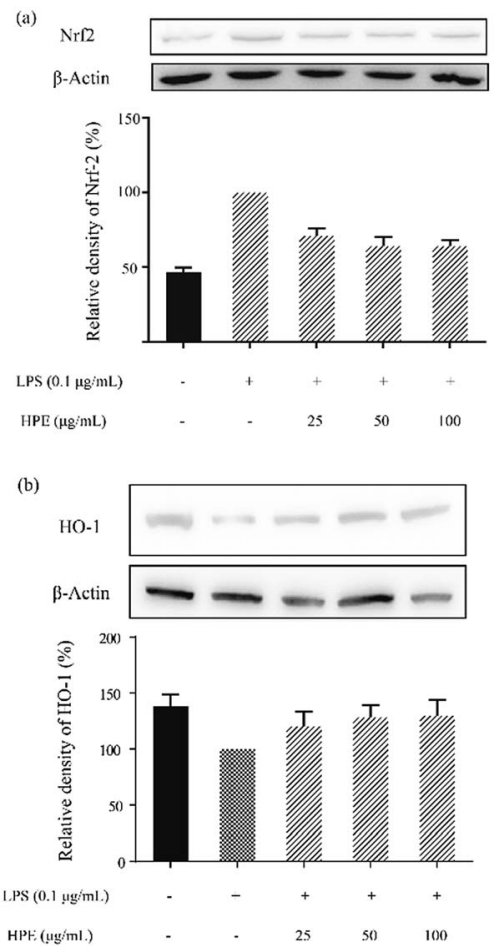

Fig. 3 Effect of huangjiu polyphenol extract (HPE) on the protein expression of (a) Nrf2 and (b) HO-1 in LPS stimulated RAW264.7 macrophages.

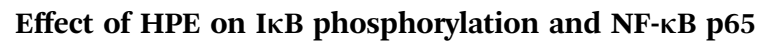
translocation in LPS stimulated RAW264.7 macrophages

In this study, the level of phosphorylated $\mathrm{I} \kappa \mathrm{B}(\mathrm{p}-\mathrm{I} \kappa \mathrm{B})$ was analysed by WB and the results are shown in Fig. 4a. After stimulation by LPS, an increase in p-IкB level was observed. HPE treatment down-regulated the $\mathrm{p}-\mathrm{I} \kappa \mathrm{B}$ level and exhibited a dosedependent inhibitory effect. Meanwhile, the translocation level of the NF- $\kappa$ B subunit, p65, was also down-regulated after being treated with HPE (Fig. 4b).

Effects of HPE on the phosphorylation of proteins in the MAPK pathway

To further confirm the anti-inflammatory pathway, the effects of HPE on the expression of phosphorylated proteins in the MAPK pathway were studied. Western blotting was carried out to evaluate the expression of p38, Erk 1/2, and JNK and the phosphorylated (P-) proteins, including P-p38, P-Erk 1/2, and PJNK. The results showed that the P-p38, P-Erk 1/2, and P-JNK levels were down-regulated by the treatment of HPE in the LPS-stimulated RAW264.7 macrophages (Fig. 5).

\section{Discussion}

As a traditional alcoholic beverage, the unique process and various raw materials make huangjiu (Chinese rice wine) contain various functional and nutritional components. Among these components, the polyphenols in huangjiu are related to many biological activities, including antioxidant activity, ${ }^{19}$ anti- 


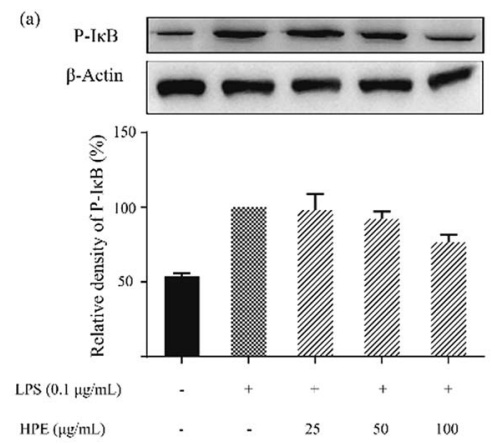

(b)
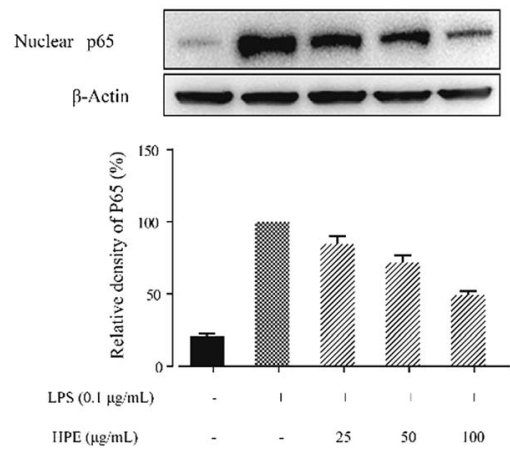

Fig. 4 Effect of huangjiu polyphenol extract (HPE) on (a) $1 \kappa B$ phosphorylation and (b) the translocation of NF- $\mathrm{BB}$ p65 in LPS stimulated RAW264.7 macrophages.
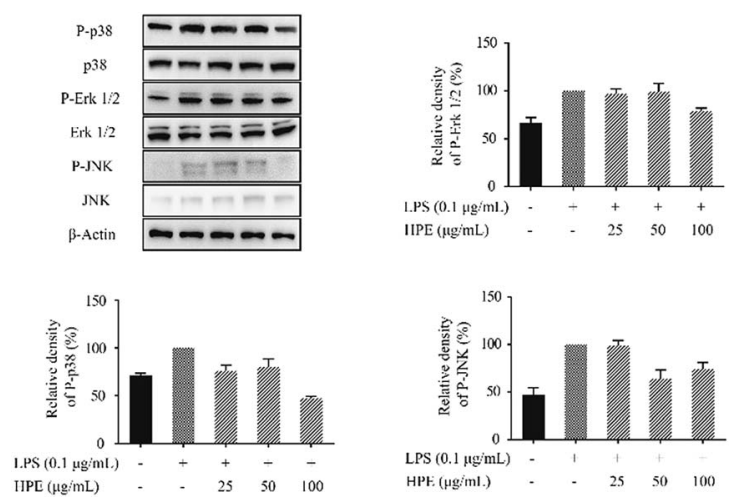

Fig. 5 Effect of huangjiu polyphenol extract (HPE) on the expression of P-p38, P-Erk 1/2 and P-JNK in LPS-stimulated RAW264.7 macrophages.

atherosclerotic activity ${ }^{23,24}$ and the prevention of cardiovascular disease. ${ }^{22}$ Solid phase extraction (SPE) is commonly used to extract polyphenols for further quantification and identification. However, this method has some disadvantages, as it is time-consuming and leads to a very low yield of polyphenols in huangjiu extraction (data not shown). Liquid-liquid extraction with ethyl acetate was also used for polyphenol extraction in this study, and the results indicated that only $12.83 \%$ polyphenols in huangjiu were detected in the organic phase (ESI Table S2 $†$ ). Moreover, crude polyphenols also contain monosaccharides, oligosaccharides, peptides and amino acids, which could interfere with further quantification and identification in
HPLC. Therefore, a method is necessary to effectively extract polyphenols from huangjiu for further biological activity studies.

In consideration of this situation, we established a method combining extraction and purification to better extract polyphenol from huangjiu. After extraction and purification, $22.57 \%$ polyphenols from huangjiu were successfully extracted, and most of the proteins and sugars were removed from huangiu polyphenol extract (HPE), which was used for further HPLC analysis and anti-inflammatory experiments. This established method is suitable for polyphenol extraction at a large scale and its application will supply sufficient samples for biological activities analysis.

Using this method, HPE with low levels of total protein and total sugar was used for further HPLC analysis. The results indicated that HPE contains 11 polyphenols and (+)-catechin is the predominant phenolic compound among the 11 detected polyphenols. This result is in accordance with previous research, which reported the highest content of $(+)$-catechin among all detected polyphenols in commercially available huangjiu..$^{30,31}$ In previous studies, (+)-catechin exhibited various bioactivities, and polyphenol extract with a high content of $(+)$-catechin also showed bioactive potential, including antioxidant, antibacterial and anti-inflammatory properties. ${ }^{32-34}$ Therefore, HPE, which is rich in the phenolic compound $(+)$-catechin, may be a potential agent for anti-inflammatory applications.

The murine macrophage cell line RAW264.7 is frequently used as the model cell for inflammation-related research due to its reproducible response to LPS or TNF- $\alpha$. In this study, LPSstimulated RAW264.7 macrophages were successfully established to test the anti-inflammatory activity of HPE. The results indicated that the production of $\mathrm{NO}$ and pro-inflammatory cytokines (e.g. IL-1 $\beta$, TNF- $\alpha$, IL-6) was effectively inhibited by HPE. In RAW264.7 cells, NO is mainly produced by inducible nitric oxide synthase (iNOS), which is regulated by heme oxygenase-1 (HO-1), an anti-inflammatory enzyme. ${ }^{35}$ HO- 1 is mainly regulated by the transcription factor Nrf2, which translocates to the nucleus and activates the expression of HO-1. In previous studies, polyphenol extracts inhibited NO production by up-regulating the translocation of Nrf2 into the nucleus and thus enhanced HO-1 expression, which finally inhibited NO production. ${ }^{26,27}$ In this study, the treatment of HPE in LPSstimulated RAW264.7 cells led to the up-regulation of HO-1 expression and Nrf2 translocation, and thus led to the downregulation of iNOS expression. Therefore, this mechanism explains the inhibitory effect of HPE on NO production in LPSstimulated RAW264.7 cells.

Pro-inflammatory cytokine expression is also regulated by HO-1 expression and Nrf2 translocation. ${ }^{26,27}$ However, proinflammatory cytokine expression may be regulated by more than one pathway. As well as Nrf2, NF- $\mathrm{BB}$ is also an important transcription factor in the inflammation process and regulates the expression of cytokines, chemokines and growth factors. ${ }^{36}$ NF-KB was first found in the B cell nucleus and reported by Sen \& Baltimore. ${ }^{37}$ In the following years, NF- $\kappa B$ was found to play a central role in immunological processes. NF- $\mathrm{B}$ is 
constitutively located in the cytoplasm in the inactive form, which interacts with its inhibitory protein $\mathrm{I} \kappa \mathrm{B} .{ }^{38}$ The activation of NF- $\kappa$ B is carried out by the stimulation of cells by LPS or some inflammatory cytokines. During the process of NF- $\kappa \mathrm{B}$ activation, $\mathrm{I} \kappa \mathrm{B}$ is phosphorylated and subsequently degraded, while the released $\mathrm{NF}-\kappa \mathrm{B}$ dimers (p65 and p50) move into the nucleus. After binding to the target location in DNA, NF- $\kappa \mathrm{B}$ initiates the transcription of the target genes and promotes the production of pro-inflammatory cytokines. Some pro-inflammatory cytokines also stimulate cells to activate NF- $\mathrm{B}$, and thus act as autocrine feedback signals to further enhance the production of these cytokines. In this study, treatments of HPE effectively inhibit the phosphorylation of $\mathrm{I} \kappa \mathrm{B}$ and also show the downtrend of p65 (subunit of NF- $\kappa \mathrm{B}$ ) levels in the nucleus. Therefore, HPE suppresses pro-inflammatory cytokine production through the NF- $\kappa \mathrm{B}$ pathway.

Recently, numerous studies have revealed that the MAPK pathway is also involved in anti-inflammatory activity. ${ }^{26,39}$ MAPK family proteins, including p-38, Erk $1 / 2$ and JNK, participate in the inflammation with their phosphorylated forms, e.g. P-p-38, P-Erk 1/2, and P-JNK. The phosphorylation of the MAPK family proteins promotes the activation and translocation of $\mathrm{NF}-\kappa \mathrm{B}$, and thus promotes pro-inflammatory cytokine expression. Our results indicated that the process of phosphorylation stimulated by LPS is inhibited by HPE treatment. Therefore, we proposed the possible mechanism of HPE exerting antiinflammatory activity including the inactivation of $\mathrm{NF}-\kappa \mathrm{B}$ (by inhibiting the phosphorylation of I $\mathrm{B}$ and NF- $\kappa \mathrm{B}$ translocation to the nucleus), the phosphorylation of the MAPK family proteins (p-38, Erk1/2 and JNK) and the activation of Nrf2 and HO-1.

\section{Conclusion}

Based on the established extraction method, huangjiu polyphenol extract (HPE) was obtained and HPLC analysis indicated that the predominant polyphenol is (+)-catechin. In LPSstimulated RAW264.7 macrophages, HPE exhibited great antiinflammatory activity and effectively inhibited the expression of NO and pro-inflammatory cytokines. Moreover, we proposed the possible mechanism of the HPE anti-inflammatory activity, which will be beneficial for gaining a better understanding of the potential value of HPE. From this study, we revealed the functional and nutritional values of huangjiu, which have potential for the prevention of inflammatory-associated diseases by the suitable uptake of polyphenols.

\section{Conflicts of interest}

There are no conflicts to declare.

\section{Acknowledgements}

This work was financially supported by the National Key Research and Development Program of China (No. 2016YFD0400504, 2017YFD0400103 and 2018YFD0400401), the National Natural Science Foundation of China (No. 31771968,
31701593 and 31571823), the National First-Class Discipline Program of Light Industry Technology and Engineering (No. LITE2018-13), and the Free Exploration Funding Project of the State Key Laboratory of Food Science and Technology in Jiangnan University (No. SKLF-ZZB-201804).

\section{References}

1 J. Zhou, Chinese Rice Wine Brewing Process, China Light Industry Press, Beijing, 1996.

2 F. Shen, D. Yang, Y. Ying, B. Li, Y. Zheng and T. Jiang, Food Bioprocess Technol., 2012, 5, 786-795.

3 H. Cai, J. Huang, G. Xu, Z. Yang, M. Liu, Y. Mi, W. Liu, H. Wang and D. Qian, PLoS One, 2012, 7, e36936.

4 J. Xiao, J. P. Huang, G. F. Xu, D. X. Chen, G. Y. Wu, M. Zhang, Y. Shen and H. Cai, Nutr. Metab., 2015, 12, 1-12.

5 C. Shen, J. Mao, Y. Chen, X. Meng and Z. Ji, J. Sci. Food Agric., 2015, 95, 1991-1996.

6 F. L. Han and Y. Xu, J. Inst. Brew., 2012, 117, 238-250.

7 S. Fei, X. Y. Niu, D. T. Yang, Y. B. Ying, B. B. Li, G. Q. Zhu and W. Jian, J. Agric. Food Chem., 2010, 58, 9809-9816.

8 F. Shen, J. Wu, Y. Ying, B. Li and T. Jiang, Food Chem., 2013, 141, 4026-4030.

9 Q. Y. Lu, R. P. Lee, J. Huang, J. Yang, S. M. Henning, X. Hong, D. Heber and Z. Li, J. Food Compos. Anal., 2015, 44, 86-92.

10 L. Chen, H. Huang, W. Liu, N. Peng and X. Huang, J. Agric. Food Chem., 2010, 58, 3507-3511.

$11 \mathrm{X}$. Mo and Y. Xu, J. Inst. Brew., 2012, 116, 304-311.

12 E. Xu, Z. Wu, J. Long, F. Wang, X. Xu, Z. Jin and A. Jiao, J. Funct. Foods, 2015, 17, 214-226.

13 Q. Fei and M. X. Pan, Food Res. Int., 2006, 39, 581-587.

14 S. Chen and Y. Xu, J. Inst. Brew., 2013, 119, 71-77.

15 C. Manach, A. Scalbert, C. Morand, C. Rémésy and L. Jiménez, Am. J. Clin. Nutr., 2004, 79, 727-747.

16 Y. Huang, W. W. Lu, B. Chen, M. Wu and S. G. Li, Food Analytical Methods, 2015, 8, 825-832.

17 Y. Wang, Y. Liu, C. Xiao, L. Liu, M. Hao, J. Wang and X. Liu, J. Food Sci., 2014, 79, C1100-C1105.

18 K. Z. Zhang, K. Deng, H. B. Luo, J. Zhou, Z. Y. Wu and W. X. Zhang, J. Inst. Brew., 2013, 119, 182-190.

19 A. Anandhan, K. Tamilselvam, T. Radhiga, S. Rao, M. M. Essa and T. Manivasagam, Brain Res., 2012, 1433, 104-113.

20 L. M. Wang, Y. J. Wang, M. Cui, W. J. Luo, X. J. Wang, P. A. Barber and Z. Y. Chen, Eur. J. Neurosci., 2013, 37, 1669-1681.

21 T. Sathyapalan, S. Beckett, A. S. Rigby, D. D. Mellor and S. L. Atkin, Nutr. J., 2010, 9, 55.

22 H. Guo, L. Liu, Y. Shi, A. Sun, F. Xu, J. Chi and D. Huang, Cardiovasc. Ther., 2010, 28, 161-168.

23 X. Zhai, J. Chi, W. Tang, Z. Ji, F. Zhao, C. Jiang, H. Lv and H. Guo, J. Pharmacol. Sci., 2014, 125, 132-141.

24 L. Meng, H. Lin, S. Pan, W. Tang, C. Zhou, X. Zhai, C. Jiang, Z. Ji, J. Chi, F. Peng and H. Guo, Int. J. Clin. Exp. Med., 2016, 9, 17539-17548.

25 L. Meng, L. Liu, C. Zhou, S. Pan, X. Zhai, C. Jiang, Y. Guo, Z. Ji, J. Chi and F. Peng, J. Cardiovasc. Pharmacol., 2016, 67, 482-490. 
26 J. T. Lin, Y. Y. Chang, Y. C. Chen, B. Y. Shen and D. J. Yang, Food Funct., 2017, 8, 1245.

27 Q. Hu, B. Yuan, H. Xiao, L. Zhao, X. Wu, K. Rakariyatham, L. Zhong, Y. Han, B. K. Muinde and W. Yang, Food Funct., 2018, 9, 1601-1611.

28 Y. Shen, C. Hu, H. Zhang and J. Hao, J. Food Biochem., 2018, 42, e12488.

29 R. M. Lamuela-Raventós, V. L. Singleton and R. Orthofer, Analysis of Total Phenols and Other Oxidation Substrates and Antioxidants by Means of Folin-Ciocalteu Reagent, John Wiley \& Sons, 1999.

30 Q. Fei and M. X. Pan, Food Res. Int., 2006, 39, 581-587.

31 Y. Huang, W. W. Lu, B. Chen, M. Wu and S. G. Li, Food Analytical Methods, 2015, 8, 825-832.

32 J. H. Kwon, S. B. Kim, K. H. Park and M. W. Lee, Arch. Pharmacal Res., 2011, 34, 1459-1466.
33 X. Li, J. Y. Zhang, W. Y. Gao, Y. Wang, H. Y. Wang, J. G. Cao and L. Q. Huang, J. Agric. Food Chem., 2012, 60, 8738-8744.

34 J. C. Liao, J. S. Deng, C. S. Chiu, S. S. Huang, W. C. Hou, W. C. Lin and G. J. Huang, Am. J. Chin. Med., 2012, 40, 1047-1062.

35 M. John, Q. Xie and C. Nathan, Annu. Rev. Immunol., 1997, 15, 323-350.

36 S. Bindu, S. Mazumder, S. Dey, C. Pal, M. Goyal, A. Alam, M. S. Iqbal, S. Sarkar, S. A. Azhar and C. Banerjee, Free Radical Biol. Med., 2013, 65, 456-467.

37 R. Sen and D. Baltimore, Cell, 1986, 46, 705-716.

38 J. A. S. Baldwin, Annu. Rev. Immunol., 1996, 14, 649-681.

39 H. P. Syama, T. Sithara, S. L. Krishnan and P. Jayamurthy, J. Funct. Foods, 2018, 44, 218-226. 